\title{
Learning One-Variable Pattern Languages in Linear Average Time
}

\author{
Rüdiger Reischuk* \\ Med. Universität zu Lübeck \\ Institut für Theoretische Informatik \\ Wallstraße 40 \\ 23560 Lübeck, Germany \\ reischuk@informatik.mu-luebeck.de
}

\author{
Thomas Zeugmann \\ Department of Informatics \\ Kyushu University \\ Kasuga 816-8580 \\ Japan \\ thomas@i.kyushu-u.ac.jp
}

\begin{abstract}
A new algorithm for learning one-variable pattern languages is proposed and analyzed with respect to its average-case behavior. We consider the total learning time that takes into account all operations till an algorithm has converged to a correct hypothesis. For the expectation it is shown that for almost all meaningful distributions defining how the pattern variable is replaced by a string to generate random examples of the target pattern language this algorithm converges within a constant number of rounds with a total learning time that is linear in the pattern length. Thus, the algorithm is average-case optimal in a strong sense.

Though one-variable pattern languages cannot be inferred finitely, our approach can also be considered as probabilistic finite learning with high confidence.
\end{abstract}

\section{INTRODUCTION}

The formal definition of patterns and pattern languages goes back to Angluin [1]. Since then, pattern languages and variations thereof have been widely investigated (cf., e.g., [12, 13, 15].

As far as learning theory is concerned, pattern languages are a prominent example of nonregular languages that can be learned in the limit from positive data. The corresponding learning model goes back to Gold [5]. Let $L$ be any language; then a text for $L$ is any infinite sequence of strings containing eventually all strings of $L$,

\footnotetext{
${ }^{*}$ This work was performed while this author was visiting the Department of Informatics at Kyushu University and was supported by the Japan Society for the Promotion of Science under Grant JSPS 29716102.
}

and nothing else. The information given to the learner are successively growing initial segments of a text. Processing these segments, the learner has to output $h y$ potheses about $L$. The hypotheses are chosen from a prespecified set called hypothesis space. The sequence of hypotheses has to converge to a correct description of the target language.

Looking at applications of limit learners, efficiency becomes a central issue. But defining an appropriate measure of efficiency for learning in the limit is a difficult problem (cf. [10]). Various authors have studied the efficiency of learning in terms of the update time needed for computing a new single hypothesis. But what counts in applications is the overall time needed by a learner until convergence, i.e., the total learning time. Since the total learning time is unbounded in the worst-case, we study the expected total learning time. Next, we shortly summarize what has been known in this regard.

Angluin [1] provides a learner for the class of all pattern languages that is based on the notion of descriptive patterns. Here a pattern $\pi$ is said to be descriptive (for the set $S$ of strings contained in the input provided so far) if $\pi$ can generate all strings contained in $S$ and no other pattern having this property generates a proper subset of the language generated by $\pi$. Since no efficient algorithm is known for computing descriptive patterns, and finding a descriptive pattern of maximum length is $\mathcal{N} \mathcal{P}$-hard, its update time is practically infeasible.

Therefore, one has considered restricted versions of pattern language learning in which the number $k$ of different variables is fixed, in particular the case of a single variable. Angluin [1] gives a learner for one-variable pattern languages with update time $O\left(\ell^{4} \log \ell\right)$, where $\ell$ is the sum of the length of all examples seen so far. Nothing is known concerning the expected total learning time of her algorithm.

Erlebach et al. $[3,4]$ have presented a one-variable pattern learner achieving an average total learning time $O\left(|\pi|^{2} \log |\pi|\right)$, where $|\pi|$ is the length of the target pattern. This result is also based on finding descriptive patterns quickly. While this approach has the advantage that the descriptiveness of every hypothesis output is guaranteed, it may have the disadvantage of preventing the learner to achieve a better expected total learning 
time. Thus, we ask whether there is a one-variable pattern language learner achieving a subquadratic expected total learning time. Clearly, the best one can get is a linear average total learning time. If this is really possible, then such a learner seems to be more appropriate for potential application than previously obtained ones, even if there are no guaranteed properties concerning the intermediately calculated hypotheses. Such a learner would have already finished his learning task with high probability before any of the known learner has computed a single guess.

What we like to present in this paper is such a onevariable pattern learner. Moreover, we prove that our learner achieves an expected linear total learning time for a very large class of distributions with respect to which the input examples are drawn.

\section{PRELIMINARIES}

Let $\mathbb{N}=\{0,1,2, \ldots\}$ be the set of all natural numbers, and let $\mathbb{N}^{+}=\mathbb{N} \backslash\{0\}$. For all real numbers $y$ we define $\lfloor y\rfloor$, the floor function, to be the greatest integer less than or equal to $y$. Let $\Sigma$ be an alphabet with $s:=|\Sigma| \geq 2$. By $\Sigma^{*}$ we denote the free monoid over $\Sigma$, and we set $\Sigma^{+}=\Sigma^{*} \backslash\{\varepsilon\}$, where $\varepsilon$ is the empty string. Let $x$ be a symbol with $x \notin \Sigma$. Every string over $(\Sigma \cup\{x\})^{+}$is called a one-variable pattern. We refer to $x$ as the pattern variable. Pat denotes the set of all one-variable patterns. We write $\#(\pi, x)$ for the number of occurrences of the pattern variable $x$ in $\pi$.

The length of a string $w \in \Sigma^{*}$ and of a pattern $\pi \in$ Pat is denoted by $|w|$ and $|\pi|$, respectively. Let $w$ be a string with $\ell=|w| \geq 1$, and let $i \in\{1, \ldots, \ell\}$; we use $w[i]$ and $w[-i]$ to denote the $i$-th symbol in $w$ counted from left to right and right to left, respectively, i.e.,

$$
\begin{aligned}
w & =w[1] w[2] \ldots w[\ell-1] w[\ell] \\
& =w[-\ell] w[-\ell+1] \ldots w[-2] w[-1] .
\end{aligned}
$$

For $1 \leq i \leq j \leq \ell$ we denote the substring $w[i] \ldots w[j]$ of $w$ by $w[i \ldots j]$. Let $\pi \in P a t$ and $u \in \Sigma^{+}$; we use $\pi[x / u]$ for the string $w \in \Sigma^{+}$obtained by substituting all occurrences of $x$ in $\pi$ by $u$. The string $u$ is called a substitution. For every $\pi \in$ Pat we define the language generated by pattern $\pi$ by

$$
L(\pi):=\left\{y \in \Sigma^{+} \mid \exists u \in \Sigma^{+}, \quad y=\pi[x / u]\right\}
$$

For discussing our approach to learning all one-variable pattern languages we let

$$
\pi=w_{0} x^{\alpha_{1}} w_{1} x^{\alpha_{2}} w_{2} \ldots w_{m-1} x^{\alpha_{m}} w_{m}
$$

be the target pattern throughout this paper. Here the $\alpha_{i}$ denote positive integers (the multiplicity by which $x$ appears in a row), and $w_{i} \in \Sigma^{*}$ the separating constant substrings, where for $1 \leq i<m$ the $w_{i}$ are assumed to be nonempty.

The learning problem considered in this paper is exact learning in the limit from positive data. A sequence $\left(\psi_{i}\right)_{i \in \mathbb{N}^{+}}$of patterns is said to converge to a pattern $\pi$ if $\psi_{i}=\pi$ for all but finitely many $i$.
Definition 1. Given a target pattern $\pi$, the learner gets a sequence of example strings $X_{1}, X_{2}, \ldots$ from $L(\pi)$. Having received $X_{g}$ he has to compute as hypothesis a one-variable pattern $\psi_{g}$. The sequence of guesses $\psi_{1}, \psi_{2}, \ldots$ eventually has to converge to a pattern $\psi$ such that $L(\psi)=L(\pi)$.

Note that in the case of one-variable pattern languages this implies that $\psi=\pi$. Some more remarks are mandatory here. Though our definition of learning resembles that one given in Gold [5], there is also a major difference. In [5] the sequence $\left(X_{i}\right)_{i \in \mathbb{N}^{+}}$is required to exhaust $L(\pi)$ in the limit, that is to fulfill $\left\{X_{i} \mid i \in \mathbb{N}^{+}\right\}=L(\pi)$. Nevertheless, in real applications this requirement will be hardly fulfilled. We therefore omit this assumption here. Instead, we only require the sequence $\left(X_{i}\right)_{i \in \mathbb{N}}$ to contain "enough" information to recognize the target pattern $\pi$. What is meant by "enough" will be made precise when discussing the set of all admissible distributions with respect to which the example sequences are allowed to be randomly drawn.

We continue with the complexity measure considered in this paper. The length of the pattern $\pi$ to be learned is given by $n:=n_{w}+n_{x}$ with $n_{w}:=\sum\left|w_{i}\right|$ and $n_{x}:=\sum \alpha_{i}$. This parameter will be considered as the size of problem instances, and the complexity analysis will be done with respect to this value $n$. We assume the same model of computation and the same representation of patterns as Angluin [1], i.e., in particular a random access machine that performs a reasonable menu of operations each in unit time on registers of length $O(\log n)$ bits, where $n$ is the input length. The inputs are read via a serial input device, and reading a string of length $n$ is assumed to require $n$ steps.

In contrast to previous work $[1,6,14,16]$, we measure the efficiency of a learning algorithm by estimating the overall time taken by the learner until convergence. This time is referred to as the total learning time. We aim to determine the total learning time in dependence on the length of the target pattern. Of course, if examples are provided by an adversary the number of examples one has to see before being able to converge is unbounded in general. Thus analyzing the total learning time in such a worst-case setting will not yield much insight. But such a scenario is much too pessimistic for many applications, and therefore, one should consider the average-case behavior. Analyzing the expected total learning time of limit learners has been initiated by Zeugmann [17]. Average-case complexity in general depends very much on the distribution over the input space. We perform our analysis for a very large class of distributions. An optimal result of linear expected total learning is achieved by carefully analyzing the combinatorics of words generated by a one-variable pattern. This linear bound can even be shown to hold with high probability. Let

$$
\mu: \Sigma^{+} \rightarrow[0,1]
$$

be the probability distribution specifying how given a pattern $\pi$ the variable $x$ is replaced to generate random examples $\pi[x / Z]$ from $L(\pi)$. Here $Z=Z_{\mu}$ is a 
random variable with distribution $\mu$.

$$
\operatorname{Range}(Z):=\left\{w \in \Sigma^{+} \mid \mu(w)>0\right\}
$$

denotes the range of $Z$, i.e., the set of all substitution strings that may actually occur. From this we get a probability distribution

$$
\mu_{\pi}: \Sigma^{+} \rightarrow[0,1]
$$

for the random strings generated by $\pi$ based on $\mu$. Let $X=X_{\pi, \mu}$ denote a random variable with distribution $\mu_{\pi}$. The random examples are then generated according to $X$, thus the relation between $X$ and $Z$ is given by $X=w_{0} Z^{\alpha_{1}} w_{1} Z^{\alpha_{2}} w_{2} \ldots w_{m-1} Z^{\alpha_{m}} w_{m}$. Note that $\mu$ is fixed, and in particular independent of the special target pattern to be learned.

What we consider in the following is a large class $\mathcal{D}$ of distributions $\mu$ that is defined by requiring only very simple properties. These properties basically exclude the case where only a small subset of all possible example strings occur and this subset does not provide enough information to reconstruct the pattern. We show that there exists an algorithm that efficiently learns every one-variable pattern on the average with respect to every distribution in $\mathcal{D}$.

By $\boldsymbol{E}[|\boldsymbol{Z}|]$ we denote the expectation of $|Z|$, i.e., the average length of a substitution. Then the expected length of an example string $X$ for $\pi$ is given by $E[|X|]$ $=n_{w}+n_{x} \cdot E[|Z|] \leq n \cdot E[|Z|]$. Obviously, if one wants to analyze the bit complexity of a learning algorithm with respect to the pattern length $n$ one has to assume that $E[|X|]$, and hence $E[|Z|]$, is finite, otherwise already the expected length of a single example will be infinite.

\section{Assumption 1. $\quad E[|Z|]<\infty$.}

Let $\mathcal{X}=X_{1}, X_{2}, X_{3}, \ldots$ denote a sequence of random examples that are independently drawn according to $\mu_{\pi}$. Note that the learner, in general, does not have information about $\mu_{\pi}$ a priori. On the other hand, the average-case analysis of our learning algorithm presupposes information about the distribution $\mu$. Thus, unlike the PAC-model, our framework is not completely distribution-free. Nevertheless, we aim to keep the information required about $\mu$ as small as possible. Finally, let

$$
L(\pi, \mu):=\left\{y \in \Sigma^{+} \mid \mu_{\pi}(y)>0\right\}
$$

be the language of all example strings that may actually occur.

\section{PROBABILISTIC ANALYSIS OF SUBSTITUTIONS}

For obtaining most general results we would like to put as little constraints on the distribution $\mu$ as possible. Note that one cannot learn a target pattern if only example strings of a very restricted form occur. This will be in particular the case if $\operatorname{Range}(Z)$ itself is contained in a nontrivial one-variable pattern language. For seeing this, suppose there exists a pattern $\phi \in P a t \backslash\{x\}$ such that Range $(Z) \subseteq L(\phi)$. Clearly, then the languages generated by $\pi=w_{0} x^{u_{0}} w_{1} x^{u_{1}} w_{2} \ldots w_{m-1} x^{u_{m}} w_{m}$ and $\pi^{\prime}=w_{0} \phi^{u_{0}} w_{1} \phi^{u_{1}} w_{2} \ldots w_{m-1} \phi^{u_{m}} w_{m}$ cannot be distinguished, since $L(\pi, \mu) \subseteq L\left(\pi^{\prime}\right)$. Thus, even from an information theoretic point of view the learner has no chance to distinguish this case from the one where the pattern to be learned is actually $\pi^{\prime}$ and the examples are generated by the corresponding projection $\mu^{\prime}$ of $\mu$. Hence, such a problem instance $(\pi, \mu)$ should be regarded as the instance $\left(\pi^{\prime}, \mu^{\prime}\right)$. To exclude this case, let us define

$$
p_{0}:=\max _{\phi \text { pattern, }|\phi|>1} \operatorname{Pr}[Z \in L(\phi)] .
$$

and let us make

\section{Assumption 2. $\quad p_{0}<1$.}

An alternative approach would be to consider the correctness of the hypotheses computed with respect to the distribution $\mu$. The learner solves the learning problem if he converges to a pattern $\psi$ for which $L(\psi, \mu)=L(\pi, \mu)$. This model is equivalent, but conceptually more involved and complicates the algorithm. Therefore we stick to the original definition. If $p_{0}<1$ then the following quantities

$$
\begin{aligned}
& p_{a}:=\max _{\sigma \in \Sigma} \operatorname{Pr}[Z[1]=\sigma], \\
& p_{e}:=\max _{\sigma \in \Sigma} \operatorname{Pr}[Z[-1]=\sigma],
\end{aligned}
$$

are smaller than 1, too. Otherwise, for some $\sigma$ it would hold Range $(Z) \subseteq L(\sigma x)$ or $\operatorname{Range}(Z) \subseteq L(x \sigma)$. To illustrate these quantities, consider the special situation of length-uniform distributions, i.e., distributions where the lengths $|Z|$ of the substitutions may be arbitrary, but for each length $\ell$ all possible strings over $\Sigma^{+}$of that length have the same probability. Then it is easy to see that $p_{0} \leq 1 / s$ and $p_{a}=p_{e}=1 / s$.

In general, define

$$
p:=\max \left\{p_{a}, p_{e}\right\}<1,
$$

and for sequences of substitutions $\mathcal{Z}=Z_{1}, Z_{2}, Z_{3}, \ldots$ the event

$$
\begin{aligned}
F_{g}[\mathcal{Z}] & :=\left[\left(Z_{1}[1]=Z_{2}[1]=\cdots=Z_{g}[1]\right)\right. \\
& \left.\vee \quad\left(Z_{1}[-1]=Z_{2}[-1]=\cdots=Z_{g}[-1]\right)\right] .
\end{aligned}
$$

Then $\operatorname{Pr}\left[F_{g}\right] \leq 2 p^{g-1}$.

Moreover, we define $f(\mathcal{Z}):=\min \left\{g \mid \neg F_{g}[\mathcal{Z}]\right\}$.

Lemma 1. The expectation of $f(\mathcal{Z})$ can be bounded as $E[f(\mathcal{Z})] \leq 2 /(1-p)$.

Proof. Clearly, if $m<\min \left\{g \mid \neg F_{g}[\mathcal{Z}]\right\}$ then $F_{m}[\mathcal{Z}]$ holds. Thus, we can estimate $\operatorname{Pr}[f(\mathcal{Z})>m] \leq 2 p^{m-1}$, and a simple calculation yields

$$
\begin{aligned}
E[f(\mathcal{Z})] & =\sum_{m=0}^{\infty} \operatorname{Pr}[f(\mathcal{Z})>m] \\
& \leq 2+2 \cdot \sum_{m \geq 2} p^{m-1} \\
& =\frac{2}{1-p}
\end{aligned}
$$




\section{SYMMETRY OF STRINGS}

We now come to the main technical tool that will help us to detect the pattern variable and its replacements in example strings, respectively.

Definition 2. Let $y=y[1] y[2] \ldots y[\ell] \in \Sigma^{+}$be a string of length $\ell$. If for some $k$ with $1 \leq k \leq \ell / 2$ the $k$-length prefix and suffix of $y$ are identical, that is $y[1 \ldots k]=y[\ell-k+1 \ldots \ell]$, we say that $y$ has a $\boldsymbol{k}$-symmetry $u=y[1 \ldots k]$ (or symmetry, for short).

A symmetry $u$ of $y$ is said to be the smallest symmetry if $|u|<|\hat{u}|$ for every symmetry $\hat{u}$ of $y$ with $\hat{u} \neq u$.

Definition 3. Let $u$ be a symmetry of $y$ and choose $c, d \in \mathbb{N}^{+}$maximal such that $y=u^{c} v_{0} u^{d}$, for some string $v_{0}$, i.e., $u$ is neither a prefix nor a suffix of $v_{0}$. This includes the special case $v_{0}=\varepsilon$. In this case, since $c$ and $d$ are not uniquely determined, we choose $c \geq d$ such that their difference is at most 1 . This unique representation of a string $y$ will be called factorization of $y$ with respect to $u$ or simply $\boldsymbol{u}$-factorization, and $u$ the base of this factorization.

If all occurrences of $u$ are factored out including also possible ones in $v_{0}$ one gets a representation $y=$ $u^{c_{0}} v_{1} u^{c_{1}} v_{2} \ldots v_{r} u^{c_{r}}$ with positive integers $c_{i} \quad\left(c_{0}=\right.$ $\left.c, c_{r}=d\right)$ and strings $v_{i}$ that do not contain $u$ as substring. This will be called a complete $\boldsymbol{u}-$ factorization of $y$.

Of particular interest for a string $y$ will be its symmetry of minimal length, denoted by $\boldsymbol{m l} \boldsymbol{s}(\boldsymbol{y})$, which gives rise to the minimal factorization of $y$. For technical reasons, if $y$ does not have a symmetry then we set $m l s(y):=|y|+1$. Let $\operatorname{sym}(\boldsymbol{y})$ denote the number of all different symmetries of $y$.

The following properties will be important for the learning algorithm described later.

Lemma 2. Let $k \in \mathbb{N}^{+}$and let $u, y \in \Sigma^{+}$be any two strings such that $u$ is a $k$-symmetry of $y$. Then we have

(1) $u$ is a smallest symmetry of $y$ iff $u$ itself has no symmetry.

(2) If $y$ possesses the factorization $y=u^{c} v_{0} u^{d}$ then it has $k^{\prime}$-symmetries for $k^{\prime}=2 k, 3 k, \ldots$, $\min \{c, d\} k$, too.

(3) If $u^{c} v_{0} u^{d}$ is the minimal factorization of $y$ then, for all $k^{\prime} \in\{1, \ldots, \max \{c, d\} m l s(y)\}, y$ does not have other $k^{\prime}$-symmetries.

(4) $\operatorname{sym}(y) \leq|y| / 2 m l s(y)$.

Proof. If a symmetry $u$ of a string $y$ can be written as $u=u^{\prime} v u^{\prime}$ for a nonempty string $u$ then obviously $u^{\prime}$ is a smaller symmetry of $y$. Hence, (1) follows.

Assertion (2) is obvious. If there were other symmetries in between then it is easy to see that $u$ itself must have a symmetry and thus cannot be minimal. This proves (3).
If $v_{0}$ contains $u$ as a substring there may be other larger symmetries. For this case there must be strings $v_{1}, v_{2}$ such that $y$ can be written as

$$
y=u^{c} v_{1} u^{d} v_{2} u^{c} v_{1} u^{d}
$$

where $v_{1}$ does not contain $u$ as substring. Then $y$ has an additional symmetry for $k^{\prime}=(c+d) k+\left|v_{1}\right|$. There may be even more symmetries if $v_{2}$ is of a very special form containing powers of $u$, but we will not elaborate on this further. The important thing to note is that the length of such symmetries grows at least by an additive term $k=m l s(y)$. The bound on $\operatorname{sym}(y)$ follows.

Assertion (4) of the latter lemma directly implies the simple bound

$$
\operatorname{sym}(y) \leq|y| / 2
$$

which in most cases, however, is far too large. Only strings over a single letter alphabet can achieve this bound. For particular distributions the bound is usually much better. To illustrate this, we again consider the length-uniform case. Then, the probability that a random string $y$ has a minimal symmetry of length $k$ is given by

$$
\operatorname{Pr}[m l s(y)=k]=\operatorname{Pr}[|y| \geq 2 k] \cdot s^{-k} .
$$

Furthermore, given that $m l s(y)=k$ the probability that it has at least $c$ symmetries is bounded by

$\operatorname{Pr}[\operatorname{sym}(y) \geq c \mid m l s(y)=k] \leq s^{-k \cdot 2(c-1)} \cdot \frac{1}{1-s^{-2 c+1}}$.

Thus, the probability of having at least $c$ symmetries is at most

$$
\sum_{k \geq 1} s^{-k \cdot(2 c-1)} \leq \frac{s^{-2 c+1}}{\left(1-s^{-2 c+1}\right)^{2}} .
$$

Now, we consider the expected number of symmetries. To motivate our Assumption 3, we first continue to look at the length-uniform case.

Lemma 3. In the length-uniform case

$$
E[\operatorname{sym}(Z)] \leq \frac{s}{2(s-1)^{2}} .
$$

Proof. Using the equality $\sum_{c \geq 1} c \cdot \alpha^{c}=\frac{\alpha}{(1-\alpha)^{2}}$ for $\alpha=s^{-2 k}$ in the estimation below one gets

$$
\begin{aligned}
& E[\operatorname{sym}(Z)]= \\
& \sum_{k \geq 1} \operatorname{Pr}[m l s(Z)=k] \cdot \sum_{c \geq 1} c \cdot \operatorname{Pr}[\operatorname{sym}(Z)=c \mid m l s(Z)=k] \\
& \leq \sum_{k \geq 1} s^{-k} \sum_{c \geq 1} c \cdot s^{-k 2(c-1)} \cdot \frac{1}{1-s^{-2 c+1}} \\
& \leq \sum_{k \geq 1} s^{k} \sum_{c \geq 1} c \cdot\left(s^{-2 k}\right)^{c} \cdot \frac{1}{1-s^{-1}} \\
& =\frac{1}{1-s^{-1}} \cdot \sum_{k \geq 1} s^{k} \cdot \frac{s^{-2 k}}{\left(1-s^{-2 k}\right)^{2}} \\
& \leq \frac{s}{2(s-1)} \sum_{k \geq 1} s^{-k} \\
& =\frac{s}{2(s-1)^{2}} \text {. }
\end{aligned}
$$


Thus, in this case the number of symmetries only depends on the size $s$ of the alphabet and therefore, it is independent of the length of the strings generated. Let us now estimate the total length of all factorizations of a string $y$, which can be bounded by $|y| \cdot \operatorname{sym}(y)$. For the length-uniform case,

$$
E[|Z| \cdot \operatorname{sym}(Z)] \leq E[|Z|] \cdot E[\operatorname{sym}(Z)] .
$$

can be shown, but for arbitrary distributions, we have to require

Assumption 3. $\quad E[|Z| \cdot \operatorname{sym}(Z)]<\infty$.

Remember that we already had to assume $E[|Z|]$ to be finite. Trivially, the expectation of $|Z| \cdot \operatorname{sym}(Z)$ is guaranteed to be finite if $E\left[|Z|^{2}\right]<\infty$, that means the variance of $|Z|$ is finite, but in general weaker conditions suffice.

If $0<E[|Z| \cdot \operatorname{sym}(Z)]<\infty$ then we also have $0<$ $E[\operatorname{sym}(Z)]<\infty$. Thus we can find a constant $c$ such that

$$
E[|Z| \cdot \operatorname{sym}(Z)] \leq c \cdot E[|Z|] \cdot E[\operatorname{sym}(Z)]=O(1) .
$$

Symmetries and factorizations should be computed fast; we thus show:

Lemma 4. The minimal symmetry of a string $y$ can be found in $O(|y|)$ operations.

Given the minimal symmetry, all further symmetries can be generated in linear time.

From a symmetry, the corresponding factorization can be computed in linear time as well.

Proof. To find the minimal symmetry an iterative scanning of specific bit positions of $y$ is done. Let $\ell$ denote the length of $y$.

\section{Algorithm 1.}

For $j=1,2, \ldots, \ell-1$, we will construct subsets $I_{j}$ of $[1 \ldots \ell]$ with the property:

$$
t \in I_{j} \Longleftrightarrow y[t \ldots t+j-1]=y[1 \ldots j] .
$$

The sets are initialized with $I_{j}=\{1\}$ for all $j$. Then $I_{1}:=\{t \mid y[t]=y[1]\}$.

Assume that $I_{j-1}$ has been constructed.

$$
\text { if } \begin{aligned}
j \in I_{j-1} \text { then } \\
y[j \ldots 2 j-2]=y[1 \ldots j-1] \text { implying } \\
y[1 \ldots 2 j-2]=y[1 \ldots j-1]^{2} . \\
\forall t \in I_{j-1}: \\
\text { if } t+j-1 \in I_{j-1} \text { then } t \hookrightarrow I_{2 j-2} \\
\text { if } 2 j-1 \geq \ell \text { then stop and output FAILURE } \\
\text { else } j:=2 j-1
\end{aligned}
$$

if $j \notin I_{j-1}$ then

$\forall t \in I_{j-1}:$

if $y[t+j-1]=y[j] \quad$ then $t \hookrightarrow I_{j}$

if $\ell-j+1 \in I_{j}$ then

stop with success and output $y[1 \ldots j]$

if $j \geq \ell$ then stop and output FAILURE

$$
\text { else } j:=j+1
$$

It can be shown that this procedure considers each bit position $y[j]$ at most a logarithmic number of times from which the bound $O(\ell \log \ell)$ follows easily. For most strings, however, the complexity is linear since more than linear time is needed only for strings of highly regular structure.

\section{Algorithm 2.}

The second, and in the worst-case more efficient algorithm first computes a maximal overlap of $y$, that is a substring $w$ of maximal length such that

$$
y=w \nu_{1}=\nu_{2} w
$$

for some nonempty strings $\nu_{1}, \nu_{2}$. If $|w| \leq|y| / 2$ then $y$ can be written in the form

$$
y=w \nu w
$$

for some string $\nu$, that means $w$ is a symmetry of $y$. Since $w$ was chosen maximal it is even the maximal symmetry. If $|w|>|y| / 2$ then in the representation $y=w \nu_{1}=\nu_{2} w$ the string $w$ overlaps with itself, thus it cannot be used as a symmetry. However, let $\kappa$ denote the length of the $\nu_{i}, r=\ell-\kappa$ the length of $w$, and $r^{\prime}:=\ell \bmod \kappa$. Then

$$
w[\kappa+1 \ldots r]=w[1 \ldots r-\kappa],
$$

which implies in particular $w[1 \ldots \kappa]=w[\kappa+1 \ldots 2 \kappa]$. In the same way, $w[\kappa+1 \ldots 2 \kappa]=w[2 \kappa+1 \ldots 3 \kappa]$, thus $w$ can be written as

$$
w=w[1 \ldots \kappa]^{\lfloor r / \kappa\rfloor} w\left[1 \ldots r^{\prime}\right]
$$

and

$$
y=w[1 \ldots \kappa]^{\lfloor r / \kappa\rfloor+1} w\left[1 \ldots r^{\prime}\right]
$$

where $w\left[1 \ldots r^{\prime}\right]$ is empty for $r^{\prime}=0$. Now define

$$
w_{0}:= \begin{cases}w, & \text { if }|w| \leq \ell / 2 \\ w[1 \ldots \kappa], & \text { if } r^{\prime}=0 \\ w\left[1 \ldots r^{\prime}\right], & \text { otherwise }\end{cases}
$$

Note that $w_{0}$ is a symmetry of $y$. As already mentioned it is the maximal one in the first case, whereas in the other cases the maximal one is $w[1 \ldots \kappa]^{\ell / 2 \kappa}$ if $r^{\prime}=0$, resp. $w[1 \ldots \kappa]^{(\ell-\kappa) / 2 \kappa} w\left[1 \ldots r^{\prime}\right]$ for $r^{\prime}>0$. Symmetries of size between $w_{0}$ and the maximal one are of the form $w[1 \ldots \kappa]^{L} w\left[1 \ldots r^{\prime}\right]$ for some $1 \leq L<$ $\ell / 2 \kappa$. Having obtained $w_{0}$, iteratively in the same way we first compute the maximal overlap of this string and from this a substring $w_{1}$, and so forth until for the first time $w_{j}$ has zero overlap. Then $w_{j}$ is the minimal symmetry of $y$.

A maximal overlap (sometimes also called a maximal border) can be computed in linear time, see for example [2], chapter 3.1, where an algorithm of complexity $2|y|-3$ is described.

Given the maximal overlap, the string $w_{0}$ can easily be obtained in a linear number of steps. Since for all $j$ the length of $w_{j}$ is at most half the length of $w_{j-1}$ the whole iterative procedure stays linearly bounded.

Once we have found a symmetry $u$, computing the complete $u$-factorization of $y$ is just a simple pattern 
matching of $u$ against $y$, which can be done by well established methods in linear time.

From a complete minimal factorization based on $u_{1}$ other symmetries can be deduced by checking powers of $u_{1}$ and the equality of substrings between these powers. This can be done in a linear number of operations.

Let $\Sigma_{\text {sym }}^{+}$denote the set of all strings in $\Sigma^{+}$that possess a symmetry and let

$$
p_{\text {sym }}:=\operatorname{Pr}\left[Z \in \Sigma_{\text {sym }}^{+}\right] .
$$

We require that the distribution is not restricted to substitutions with symmetries - with positive probability also nonsymmetric substitutions should occur.

Assumption 4. $\quad p_{\text {sym }}<1$.

Now consider the event

$$
Q_{g}[\mathcal{Z}]:=\left[\left\{Z_{1}, \ldots, Z_{g}\right\} \in \Sigma_{\text {sym }}^{+}\right] .
$$

$Q_{g}[\mathcal{Z}]$ means that among the first $g$ substitutions all have a symmetry. Obviously,

$$
\operatorname{Pr}\left[Q_{g}[\mathcal{Z}]\right] \leq p_{\mathrm{sym}}^{g}
$$

Define $q(\mathcal{Z}):=\min \left\{g \mid \neg Q_{g}[\mathcal{Z}]\right\}$. Similarly to Lemma 1, one can show

Lemma 5. $E[q(\mathcal{Z})] \leq 1 /\left(1-p_{\text {sym }}\right)$.

\section{BASIC SUBROUTINES: FACTOR- IZATIONS AND COMPATIBILITY}

For a subset $A$ of $\Sigma^{*}$ let $\operatorname{PRE}(A)$ and $\operatorname{SUF}(A)$ denote the maximal common prefix and suffix of all strings in $A$, respectively. Let $m_{\text {pre }}(A)$ and $m_{\text {suf }}(A)$ be their lengths. The first goal of the algorithm is to recognize the prefix $w_{0}$ and suffix $w_{m}$ before the first and last occurrence of the variable $x$, respectively, in the pattern $\pi$. In order to avoid confusion, $x$ will be called the pattern variable, where variable simply refers to any data variable used by the learning algorithm.

The current information about the prefix and suffix is stored in the variables PRE and SUF. The remaining pattern learning is done with respect to the current value of these variables. If the algorithm sees a new string $X$ such that $\operatorname{PRE}(\{X, \mathrm{PRE}\}) \neq \mathrm{PRE}$ or $\operatorname{SUF}(\{X, \mathrm{SUF}\}) \neq \mathrm{SUF}$ then these variables will be updated. We will call this the begin of a new phase.

Definition 4. For a string $Y \in \Sigma^{+}$a (PRE, SUF) factorization is defined as follows. $Y$ has to start with prefix PRE and end with suffix SUF. For the remaining middle part $Y^{\prime}$ we select a symmetry $u_{1}$. This means $Y$ can be written as $Y=\operatorname{PRE} u_{1}^{c_{1}} v_{1} u_{1}^{d_{1}} \mathrm{SUF}$ for some strings $u_{1}, v_{1}$ and $c_{1}, d_{1} \in \mathbb{N}^{+}$.

If such a representation is not possible for a given pair (PRE, SUF) then $Y$ is said to have no (PRE, SUF) -factorization.

Moreover, $Y^{\prime}$ may have other symmetries $u_{2}, u_{3}, \ldots$ giving rise to factorizations $Y=\operatorname{PRE} u_{i}^{c_{i}} v_{i} u_{i}^{d_{i}} \mathrm{SUF}$ for $c_{i}, d_{i} \in \mathbb{N}^{+}$. For simplicity, we assume that the symmetries $u_{i}$ are ordered by increasing length, in particular $u_{1}$ always denotes the minimal symmetry with corresponding minimal factorization.

Lemma 6. Let $Y=\operatorname{PRE} u_{1}^{c_{1}} v_{1} u_{1}^{d_{1}} \mathrm{SUF}$ be the minimal (PRE,SUF)-factorization of $Y$. Then, for every string $\tilde{Y}$ of the form $\tilde{Y}=\operatorname{PRE} u_{1} \tilde{v} u_{1} \mathrm{SUF}$ for some string $\tilde{v}$, the minimal (PRE, SUF)-factorization of $\tilde{Y}$ is based on $u_{1}$, too.

Proof. That $u_{1}$ gives rise to a factorization is obvious. There cannot be one of smaller length because this implies that $u_{1}$ has a symmetry and contradicts that $u_{1}$ is minimal for $Y$.

Though the following lemma is easily verified, it is important to establish the correctness of our learner presented below.

Lemma 7. Let $\pi=w_{0} x v x w_{m}$ be any pattern with $\#(\pi, x) \geq 2$, let $u \in \Sigma^{+}$, and let $Y=\pi[x / u]$. Then $Y$ has a $\left(w_{0}, w_{m}\right)$-factorization with base $u$ and its minimal $\left(w_{0}, w_{m}\right)$-factorization is based on the minimal symmetry $u_{1}$ of $u$.

The results of Lemma 4 directly translate to

Lemma 8. The minimal base for a (PRE, SUF) factorization of a string $Y$ can be computed in time $O(|Y|)$. All additional bases can be found in linear time. Given a base, the corresponding (PRE,SUF) factorization can be computed in linear time as well.

Definition 5. Two strings $Y, \tilde{Y}$ are said to be directly compatible with respect to a given pair (PRE, SUF) if from their minimal (PRE, SUF) -factorizations a single pattern $\psi=\psi(\boldsymbol{Y}, \tilde{\boldsymbol{Y}})$ can be derived from which both strings can be generated. More precisely, it has to hold:

$Y=\operatorname{PRE} u_{1}^{c_{1}} v_{1} u_{1}^{d_{1}} \mathrm{SUF}$ and

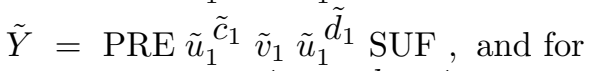

$Y_{\text {mid }}:=u_{1}^{c_{1}-1} v_{1} u_{1}^{d_{1}-1}$ and

$\tilde{Y}_{\text {mid }}:=\tilde{u}_{1}^{\tilde{c}_{1}-1} \tilde{v}_{1} \tilde{u}_{1}^{\tilde{d}_{1}-1}$ every occurrence of $u_{1}$ in $Y_{\text {mid }}$ - including further ones in $v_{1}$ - is matched in $\tilde{Y}_{\text {mid }}$ either by an occurrence of $\tilde{u}_{1}$ (which indicates that at this place $\pi$ has a pattern variable) or by $u_{1}$ itself (indicating that the constant substring $u_{1}$ occurs in $\pi$ ). In all the remaining positions $Y_{\text {mid }}$ and $\tilde{Y}_{\text {mid }}$ have to agree.

We extend this compatibility notion to pairs consisting of a string $Y$ and a pattern $\pi$. $Y$ is directly compatible to $\pi$ with respect to (PRE, SUF) if for the minimal symmetry $u_{1}$ of the (PRE, SUF)-factorization of $Y$ holds $\pi\left[x / u_{1}\right]=Y$.

The following lemma is easily verified.

Lemma 9. Assume that (PRE, SUF) $=\left(w_{0}, w_{m}\right)$ has the correct value. If a string $Y$ is generated from $\pi$ by substituting the pattern variable by a nonsymmetric string $u$ then the string $u_{1}$ on which its minimal (PRE, SUF) -factorization is based equals $u$. Thus, $Y$ is directly compatible to $\pi$. 
Proof. It is easy to see that for a nonsymmetric string $u$ the string

$Y=\pi[x / u]=w_{0} u^{\alpha_{1}} w_{1} u^{\alpha_{2}} w_{2} \ldots w_{m-1} u^{\alpha_{m}} w_{m}$

has $u$ as the basis for its minimal $\left(w_{0}, w_{m}\right)$-factorization. That $u$ gives rise to a factorization is obvious, and if there were a smaller one it would imply that $u$ has a symmetry. Since the constant substrings $w_{1}, \ldots, w_{m-1}$ may contain $u$ as a substring the actual factorization may show more powers of $u$, but it is unique since occurrences of $u$ cannot overlap - again because $u$ is nonsymmetric. If the constant substring $w_{i}$ of $\pi$ has a decomposition with respect to $u$ of the form $u^{\beta_{i, 0}} \omega_{i, 1} u^{\beta_{i, 1}} \ldots \omega_{i, n i} u^{\beta_{i, n i}}$, where the $\beta_{i, j}$ are integers and the $\omega_{i, j}$ are substrings not containing $u$, then the middle part $Y_{\text {mid }}$ of $Y$ without prefix, suffix and first and last occurrence of $u$ looks like

$$
\begin{array}{r}
u^{\alpha_{1}-1} u^{\beta_{1,0}} \omega_{1,1} u^{\beta_{1,1}} \ldots \omega_{1, n 1} u^{\beta_{1, n 1}} u^{\alpha_{2}} u^{\beta_{2,0}} \omega_{2,1} u^{\beta_{2,1}} \\
\ldots \omega_{2, n 2} u^{\beta_{2, n 2}} u^{\alpha_{3}} \ldots u^{\alpha_{m}-1} .
\end{array}
$$

When checking direct compatibility of $Y$ against $\pi$ it becomes obvious whether a substring $u$ in $Y$ corresponds to a variable or not.

If one of the substitutions $u, \tilde{u}$ for $Y=\pi[x / u]$, resp. $\tilde{Y}=\pi[x / \tilde{u}]$ is a prefix of the other, let us say $\tilde{u}=u u^{\prime}$ for some nonempty string $u^{\prime}$ then there may be an ambiguity if $u u^{\prime}$ appears as a constant substring in $Y_{\text {mid }}$. If this is not followed by another occurrence of $u^{\prime}$ it can easily be detected. In general, if $u u^{\prime}$ is a constant in $\pi$ then the number of occurrences following this substring will be the same in the corresponding positions in $Y_{\text {mid }}$ and $\tilde{Y}_{\text {mid }}$, otherwise it has to be one more in $\tilde{Y}$.

Using this observation it is easy to see that even in such a case testing of direct compatibility is easy.

Lemma 10. Let the minimal factorizations of two strings $Y, \tilde{Y}$ be given. Then by a single joint scan one can check whether they are directly compatible, and if yes construct their common pattern $\psi(Y, \tilde{Y})$. The scan can be performed in $O(|Y|+|\tilde{Y}|)$ bit operations.

Moreover, for a pattern $\pi$ it can be checked in time $O(|Y|+|\pi|)$ whether $Y$ is directly compatible to $\pi$.

The extra effort in the degenerated case of $u$ being a prefix of $\tilde{u}$ can be omitted if in this case the pattern matching is done from right to left since the procedure is completely symmetric. This will only fail if $u$ is both prefix and suffix of $\tilde{u}$, implying that $\tilde{u}=u u^{\prime} u$. But this means that $\tilde{u}$ has a symmetry and thus cannot derive from a minimal factorization of $\tilde{Y}$.

Definition 6. A string $Y$ is downwards compatible to a string $\tilde{Y}$ with respect to a given pair (PRE, SUF) if for some $\kappa \geq 1$, from the minimal (PRE, SUF)-factorization of $Y$ and the $\kappa$-th (PRE,SUF)factorization of $\tilde{Y}$ a single pattern $\psi=\psi(Y, \tilde{Y}, \kappa)$ can be derived from which both strings can be generated. We also say that $\tilde{Y}$ is upwards compatible to $Y$.
Again, these notions are extended to pairs consisting of a string and a pattern.

Lemma 11. Assume (PRE, SUF) $=\left(w_{0}, w_{m}\right)$ having the correct value. Let $Y=\pi[x / u]$ for a nonsymmetric string $u$. Any other string $\tilde{Y}$ in $L(\pi)$ obtained by substituting the pattern variable by a string $\tilde{u}$ for which $u$ is not a symmetry is upwards compatible to $Y$ with respect to (PRE, SUF) .

The pattern $\psi(Y, \tilde{Y})$ equals the pattern $\pi$ to be learned.

Given the (PRE, SUF) -factorization of both strings, $\psi(Y, \tilde{Y})$ can be constructed in time at most $O((1+$ $\operatorname{sym}(\tilde{Y})) \cdot(|Y|+|\tilde{Y}|))$, where $\operatorname{sym}(\tilde{Y}):=\operatorname{sym}(\tilde{u})$ denotes the number of symmetries of the string $\tilde{u}$ that generates $\tilde{Y}$.

Furthermore, given a pattern $\psi$ and the factorization of a string $Y$ it can be checked in time $O(|Y|+|\psi|)$ whether $Y$ is upwards compatible to $\psi$. For $Y$, downwards compatibility to $\psi$ can be checked and $\psi(Y, \psi, \cdot)$ can be constructed in linear time, too. let

Proof. Let $u$ be nonsymmetric, $Y=\pi[x / u]$, and

$$
\begin{aligned}
Y_{\text {mid }}= & u^{\alpha_{1}-1} u^{\beta_{1,0}} \omega_{1,1} u^{\beta_{1,1}} \ldots \\
& \omega_{1, n 1} u^{\beta_{1, n 1}} u^{\alpha_{2}} u^{\beta_{2,0}} \omega_{2,1} u^{\beta_{2,1}} \ldots u^{\alpha_{m}-1} .
\end{aligned}
$$

If

$$
\tilde{Y}=\pi[x / \tilde{u}]=w_{0} \tilde{u}^{\alpha_{1}} w_{1} \tilde{u}^{\alpha_{2}} w_{2} \ldots w_{m-1} \tilde{u}^{\alpha_{m}} w_{m}
$$

then $\tilde{Y}$ has a $\left(w_{0}, w_{m}\right)$-factorization based on $\tilde{u}$. Note that this factorization will not be minimal if $\tilde{u}$ itself has symmetries. Since $w_{1}, \ldots, w_{m-1}$ may contain $\tilde{u}$ the actual factorization may show more powers of $\tilde{u}$.

By assumption, $u$ is not a symmetry for $\tilde{u}$ and since one may either work from left to right or right to left we may assume that $u$ is not a prefix of $\tilde{u}$. When comparing $Y_{\text {mid }}$ to $\tilde{Y}_{\text {mid }}$ after the first $\alpha_{1}-1$ occurrences of $u$ in $Y_{\text {mid }}$ have been read and matched against occurrences of $\tilde{u}$ in $\tilde{Y}_{\text {mid }}$ the next occurrence of $u$ in the substring $u^{\beta_{1,0}}$ will be detected as a constant. This is because this substring also occurs in $\tilde{Y}_{\text {mid }}$ and $u$ is not a prefix of $\tilde{u}$. The same holds for the other occurrences of $u$ in $Y$.

Given the corresponding factorizations, checking whether $Y_{\text {mid }}$ and $\tilde{Y}_{\text {mid }}$ match can be done by a single pass over the strings and has linear time complexity. However, one has to find that factorization of $\tilde{Y}$ that matches the one of $Y$. Considering the symmetries of $\tilde{Y}$ in increasing length this will be symmetry $\operatorname{sym}(\tilde{u})$. In the worst-case, if $\tilde{u}$ contains only one symbol $\operatorname{sym}(\tilde{u})$ can be as large as $|\tilde{u}| / 2$, but such a case will be easier to handle.

This can even be sped-up. One observation is that a string with $c$ symmetries yields a least by a factor $c$ more occurrences of its minimal symmetry in the minimal factorization. Thus, once one output pattern $\psi$ has been computed, which also gives the number of occurrences of the pattern variable, strings $\tilde{Y}$ with a much 
larger number of occurrences in the minimal factorization based on a string $\tilde{u}_{1}$ can simply be discarded unless $\psi$ itself contains lots of substrings $\tilde{u}_{1}$. More precisely, let

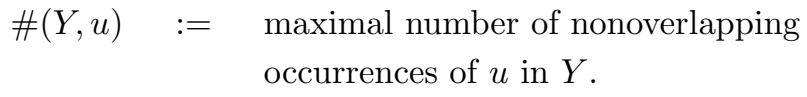

Since $u$ is nonsymmetric and $Y=\pi[x / u]$

$$
\#(Y, u)=\#(\pi, x)+\#(\pi, u) .
$$

For $\tilde{Y}=\pi[x / \tilde{u}]$ and a symmetry $\tilde{u}_{i}$ of a factorization of $\tilde{Y}$ such that $\tilde{u}_{i}$ is a substring of $\tilde{u}$ it holds,

$$
\#\left(\tilde{Y}, \tilde{u}_{i}\right)=\#\left(\tilde{u}, \tilde{u}_{i}\right) \cdot \#(\pi, x)+\#\left(\pi, \tilde{u}_{i}\right) .
$$

Let $\psi$ a pattern that is supposed to equal the pattern $\pi$ to be learned. Thus, to find the right factorization of a string $\tilde{Y}$ to check upwards compatibility against $\psi$ from the minimal factorization one can compute

$$
\frac{\#\left(\tilde{Y}, \tilde{u}_{1}\right)-\#\left(\psi, \tilde{u}_{i}\right)}{\#(\psi, x)}
$$

to get an estimate on $\#\left(\tilde{u}, \tilde{u}_{1}\right)$. When all symmetries of $\tilde{Y}$ are known it is then easy to find that string $\tilde{u}$ directly that matches this value. However, when checking upwards compatibility of a string $\tilde{Y}$ to a string $Y$, we do not have a precise estimate on $\#(\pi, x)$, there is only the upper bound $\#(Y, u)$ available from the factorization of $Y$. This implies a lower bound on $\#\left(\tilde{u}, \tilde{u}_{1}\right)$ of the form

$$
\#\left(\tilde{u}, \tilde{u}_{1}\right) \geq \frac{\#\left(\tilde{Y}, \tilde{u}_{1}\right)-\#\left(Y, \tilde{u}_{i}\right)}{\#(Y, u)} .
$$

Thus, unless \# $(\pi, u)$ is relatively large compared to $\#(\pi, x)$ this gives a good approximation which symmetry of $\tilde{Y}$ should be used.

Note that one cannot decide whether a string $Y$ was generated by substitution with a nonsymmetric string by counting the number of its factorizations - which is likely to be one. However, there are rare cases with more factorization than the one induced by the substitution for example, if $\alpha_{1}$ and $\alpha_{m}$ have a common nontrivial divisor or even if $\alpha_{1}=\alpha_{m}=1$, but by chance $w_{1}=$ $v u v^{\prime}$ and $w_{m-1}=v^{\prime \prime} u v$ for some arbitrary strings $v, v^{\prime}, v^{\prime \prime}$.

\section{THE ALGORITHM}

The learner may not store all example strings he has seen so far. Therefore let $A=A_{g}=A_{g}(\mathcal{X})$ denote the set of examples he remembers after having got the first $g$ examples of the random sequence $\mathcal{X}=X_{1}, X_{2}, \ldots$, and, similarly, let $\mathrm{PRE}_{g}$ and $\mathrm{SUF}_{g}$ be the values of the variables PRE and SUF at that time. We will call this round $g$ of the learning algorithm.

Let us first describe the global strategy of the learning procedure. When the pattern is a constant $\pi=w$ all example strings are equal to $w$ and the variables
PRE and SUF are not defined. Thus, as long as the algorithm has seen only one string, it will output this string.

Otherwise, we try to generate a pattern from 2 compatible strings received so far. If this is not possible or if one of the examples does not have a factorization then the output will be the default pattern

$$
\psi_{0}:=\mathrm{PRE}_{\boldsymbol{g}} \boldsymbol{x} \mathrm{SUF}_{\boldsymbol{g}} .
$$

If a non-default pattern has been generated as a hypothesis further examples are tested for compatibility with respect to this pattern. As long as the test is positive the algorithm will stick to this hypothesis, else a new pattern will be generated. In the simplest version of the algorithm we remember only a single example of the ones seen so far. Instead of a set $A$ we will use a single variable $Y$.

\section{The One-Variable Pattern Learning Algorithm}

$Y:=X_{1} ; \quad$ PRE $:=X_{1} ; \quad$ SUF $:=X_{1} ;$ output $X_{1}$

for $g=2,3,4, \ldots$ do

$\mathrm{PRE}^{\prime}:=\mathrm{PRE} ; \quad \mathrm{SUF}^{\prime}:=\mathrm{SUF}$;

$\psi:=$ output of previous round; read the new example $X_{g}$;

if $X_{g}=\psi$ then output $\psi$, else

$\operatorname{PRE}:=\operatorname{PRE}\left(\left\{\mathrm{PRE}, X_{g}\right\}\right)$;

$\mathrm{SUF}:=\operatorname{SUF}\left(\left\{\mathrm{SUF}, X_{g}\right\}\right) ;$

if $\mathrm{PRE} \neq \mathrm{PRE}^{\prime}$ or $\mathrm{SUF} \neq \mathrm{SUF}^{\prime}$ then

compute the (PRE, SUF) -factorization of $Y$; $\psi_{0}:=\operatorname{PRE} x \mathrm{SUF}$; $\psi:=\psi_{0}$ endif;

compute the (PRE,SUF)-factorization of $X_{g}$;

case 1: $Y$ does not have a factorization then output $\psi_{0}$ :

case 2: $X_{g}$ does not have a factorization then output $\psi_{0}$ and $Y:=X_{g}$;

case 3: $\psi=\psi_{0}$ if $X_{g}$ is downwards compatible to $Y$ then output $\psi\left(X_{g}, Y, \cdot\right)$, else output $\psi_{0}$,

if $X_{g}$ is shorter than $Y$ then $Y:=X_{g}$;

case 4: $X_{g}$ is upwards compatible to $\psi$ then output $\psi$;

case 5: $X_{g}$ is downwards compatible to $\psi$ then output $\psi\left(X_{g}, \psi, \cdot\right)$ and $Y:=X_{g}$;

else output $\psi_{0}$.

\section{PROOF OF CORRECTNESS}

Since the example strings are generated at random it might happen that only "bad examples" occur in which case no learning algorithm can eventually come up with a correct hypothesis. Therefore, the following claims cannot hold absolutely in a probabilistic setting, but they will be true with probability 1 . Remember that $\pi=w_{0} x^{\alpha_{1}} w_{1} x^{\alpha_{2}} w_{2} \ldots w_{m-1} x^{\alpha_{m}} w_{m}$ is the pattern to 
be learned. Since not all substitutions start with the same symbol or end with the same symbol (remember that we have assumed $p<1$ ) with probability 1 a sequence $\mathcal{X}$ contains strings $X_{i}, X_{j}, X_{k}$, where $X_{\kappa}=$ $\pi\left[x / u_{\kappa}\right]$ such that

$$
u_{i}[1] \neq u_{j}[1] \quad \text { and } \quad u_{i}[-1] \neq u_{k}[-1] .
$$

Note that $j$ may be equal to $k$. Let $g$ be the maximum of $i, j, k$ and consider a triple for which $g$ is minimal. By the construction of the sets PRE and SUF round $g$ will start a new phase in which now the variables $\mathrm{PRE}_{g}=w_{0}$ and $\mathrm{SUF}_{g}=w_{m}$ have the correct values.

We do not care about the output of the algorithm before this final phase has been reached. It remains to show that the algorithm will converge in the final phase. For this purpose, let us distinguish whether the pattern contains the variable only once, in which case there will be examples without any symmetry, or more than once (the case that the pattern does not contain any variable is obvious).

If $\pi=w_{0} x w_{1}$ then with probability 1 there will be an example $X_{g}$ obtained from a substitution $[x / u]$ with a nonsymmetric string $u$. Then $X_{g}$ does not have a $\left(\mathrm{PRE}_{g}, \mathrm{SUF}_{g}\right)$-factorization and thus case 2 occurs. Since $Y$ is set equal to $X$ from then on always case 1 occurs. The algorithm will always choose case 1 and output $\psi_{0}$, which in this case is the correct answer.

Otherwise, the pattern contains the variable at least twice and any example does have a $\left(\mathrm{PRE}_{g}, \mathrm{SUF}_{g}\right)$-factorization. Lemma 11 shows that a nonsymmetric substitution generates a string that is downwards compatible to any other string in $L(\pi)$. Thus, as soon as $X_{g}$ is such a string, which again happens with probability 1 , the output $\psi_{g}$ will equal the pattern $\pi$. Furthermore the algorithm will never change its output from this round on since case 4 " $X_{g^{\prime}}$ is upwards compatible to $\psi$ " will hold for any $g^{\prime}>g$.

Let us summarize these properties in the following

Lemma 12. After the algorithm has detected the correct prefix and suffix it will converge immediately to the correct hypothesis $\pi$ as soon it gets the first example generated by a nonsymmetric substitution.

\section{COMPLEXITY ANALYSIS}

Let $\psi_{g}$ denote the output of round $g$, and $Y_{g}$ the value of $Y$ at the end of that round. Let Time $_{g}(\mathcal{X})$ denote the number of bit operations in round $g$ on example sequence $\mathcal{X}$, and recall that $Z$ and $X$ are defined as random variables for the substitutions and examples, respectively.

Lemma 13. For each round $g$ it holds

$$
\begin{aligned}
E\left[\text { Time }_{g}(\mathcal{X})\right] & \leq O(E[|X|] \cdot(1+E[\operatorname{sym}(Z)])) \\
& \leq O(n \cdot E[|Z|] \cdot(1+E[\operatorname{sym}(Z)])) .
\end{aligned}
$$

Proof. By Lemma 10 and 11 in each round $g$ the number of bit operations can be estimated by

$$
\begin{gathered}
\text { Time }_{g}(\mathcal{X}) \leq \\
O\left(\left|Y_{g-1}\right|\right)+O\left(\left|X_{g}\right|\right) \\
+\quad \max \left\{O \left(\left(\left|Y_{g-1}\right|+\left|X_{g}\right|\right) \cdot\left(1+\operatorname{sym}\left(Y_{g-1}\right)\right),\right.\right. \\
O\left(\left(\left|\psi_{g-1}\right|+\left|X_{g}\right|\right) \cdot\left(1+\operatorname{sym}\left(X_{g}\right)\right),\right. \\
\left.O\left(\left|\psi_{g-1}\right|+\left|X_{g}\right|\right)\right\} \\
\leq \quad O\left(\left|Y_{g-1}\right|+\left|X_{g}\right|+\left|\psi_{g-1}\right|+\left|Y_{g-1}\right| \cdot \operatorname{sym}\left(Y_{g-1}\right)\right. \\
+\left|X_{g}\right| \cdot \operatorname{sym}\left(Y_{g-1}\right)+\left|\psi_{g-1}\right| \cdot \operatorname{sym}\left(X_{g}\right) \\
\left.+\left|X_{g}\right| \cdot \operatorname{sym}\left(X_{g}\right)\right) .
\end{gathered}
$$

By construction of the algorithm and the fact that a pattern is never longer than an example string it generates we can bound $E\left[\left|X_{g}\right|\right]$ as well as $E\left[\left|Y_{g-1}\right|\right]$ and $E\left[\left|\psi_{g-1}\right|\right]$ by $E[|X|]$. Moreover, Assumption 3 directly implies that $E\left[\left|X_{g}\right| \cdot \operatorname{sym}\left(X_{g}\right)\right]$ and $E\left[\left|Y_{g-1}\right| \cdot \operatorname{sym}\left(Y_{g-1}\right)\right]$ are both bounded by $O(E[|X|] \cdot E[\operatorname{sym}(Z)])$. Note, that $X_{g}$ is independent of $Y_{g-1}$ and $\psi_{g-1}$. Thus

$$
\begin{aligned}
E\left[\left|X_{g}\right| \cdot \operatorname{sym}\left(Y_{g-1}\right)\right] & =E\left[\left|X_{g}\right|\right] \cdot E\left[\operatorname{sym}\left(Y_{g-1}\right)\right] \\
& =E[|X|] \cdot E[\operatorname{sym}(Z)]
\end{aligned}
$$

and

$$
\begin{aligned}
E\left[\left|\psi_{g-1}\right| \cdot \operatorname{sym}\left(X_{g}\right)\right] & =E\left[\left|\psi_{g-1}\right|\right] \cdot E\left[\operatorname{sym}\left(X_{g}\right)\right] \\
& \leq E[|X|] \cdot E[\operatorname{sym}(Z)] .
\end{aligned}
$$

This simplifies the expectation to

$$
\begin{aligned}
& E\left[\text { Time }_{g}(\mathcal{X})\right] \leq \\
& O\left(E\left[\left|Y_{g-1}\right|\right]+E\left[\left|X_{g}\right|\right]+E\left[\left|\psi_{g-1}\right|\right]\right. \\
&+\quad E\left[\left|Y_{g-1}\right| \cdot \operatorname{sym}\left(Y_{g-1}\right)\right]+E\left[\left|X_{g}\right| \cdot \operatorname{sym}\left(Y_{g-1}\right)\right] \\
&+\left.E\left[\left|\psi_{g-1}\right| \cdot \operatorname{sym}\left(X_{g}\right)\right]+E\left[\left|X_{g}\right| \cdot \operatorname{sym}\left(X_{g}\right)\right]\right) \\
& \leq O(E[|X|]+E[|X|] \cdot E[\operatorname{sym}(Z)]) .
\end{aligned}
$$

Now we can also bound the total learning time.

Lemma 14. The expected total learning time is bounded by

$O\left(n \cdot E[|Z|] \cdot(1+E[\operatorname{sym}(Z)]) \cdot\left(\frac{1}{1-p}+\frac{1}{1-p_{\mathrm{sym}}}\right)\right)$.

Since $E[|Z|]), E[\operatorname{sym}(Z)], p$, and $p_{\text {sym }}$ are characterized by the distribution for substituting the pattern variable they are all independent of the problem size. This means the complexity grows linear with the size of the problem.

Proof. The number of rounds can be bounded by the number of rounds to reach the final phase plus the number of rounds in the final phase till $\psi_{g}=\pi$. By Lemma 1 and 5 the expectation of both is a constant that only depends on the probabilities $p$ and $p_{\text {sym }}$. Let $G$ be a random variable that counts the number of rounds till convergence. Then,

$$
E[G] \leq O\left(\frac{1}{1-p}+\frac{1}{1-p_{\mathrm{sym}}}\right) .
$$


Let $\operatorname{Time}_{\text {total }}(\mathcal{X})$ denote the total number of operations on example sequence $\mathcal{X}$. Then

$$
\begin{aligned}
\text { Time }_{\text {total }}(\mathcal{X}) & =\sum_{g=1}^{G} \operatorname{Time}_{g}(\mathcal{X}) \\
& =\sum_{t \geq 2} \operatorname{Pr}[G=t] \cdot \sum_{g=1}^{t} \operatorname{Time}_{g}(\mathcal{X})
\end{aligned}
$$

and

$$
\begin{gathered}
E\left[\text { Time }_{\text {total }}(\mathcal{X})\right]= \\
E\left[\sum_{t \geq 2} \operatorname{Pr}[G=t] \cdot \sum_{g=1}^{t} \operatorname{Time}_{g}(\mathcal{X})\right] \leq \\
O\left(E\left[\sum_{t \geq 2} \operatorname{Pr}[G=t] \cdot t \cdot E[|X|] \cdot(1+E[\operatorname{sym}(Z)])\right]\right) \\
\quad \leq \\
O\left(E[|X|] \cdot(1+E[\operatorname{sym}(Z)]) \cdot E\left[\sum_{t} \operatorname{Pr}[G=t] \cdot t\right]\right) \\
\quad=O(E[|X|] \cdot(1+E[\operatorname{sym}(Z)]) \cdot E[G]) \leq \\
O\left(n \cdot E[|Z|] \cdot(1+E[\operatorname{sym}(Z)]) \cdot\left(\frac{1}{1-p}+\frac{1}{1-p_{\mathrm{sym}}}\right)\right) \\
\quad=O(n) .
\end{gathered}
$$

Summarizing, we state the main result of this paper.

Theorem 1. One-variable pattern languages can be inferred in linear expected total learning time for all distributions that fulfill the Assumptions 1 through 4 made above.

Clearly, the expected value of a random variable is only one aspect of its distribution. Looking at potential applications of our learning algorithm, a hypothetical user might be interested in knowing how often the total learning time exceeds its average substantially. For answering this question we could compute the variance of the total learning time. Then Chebyshev's inequality provides the desired tail bounds. However, in our particular setting, there is an easier way to figure out how good the distribution of the total learning time is centered around its expected value. The main ingredient is the following additional nice feature of our algorithm. It convergence immediately in the final phase when an example with a nonsymmetric replacement occurs.

The expectation of this event is $E[G]$, hence with probability at least $1 / 2$ the algorithm converges within $2 E[G]$ rounds. If this did not happen, no matter which bad examples have occurred, again there will be convergence in the next $2 E[G]$ rounds with probability at least $1 / 2$. Thus, the probability of failure decreases exponentially with the number of rounds, more precisely, for all $m \in \mathbb{N}$ it holds:

$$
\operatorname{Pr}\left[\text { Time }_{\text {total }} \geq 2 \cdot m \cdot E\left[\text { Time }_{\text {total }}\right]\right] \leq 2^{-m} \text {. }
$$

Since the distribution of Time $_{\text {total }}$ decreases exponentially, all higher moments of it exist. In particular, we may conclude that the variance of Time $_{\text {total }}$ is small.

\section{CONCLUSIONS}

We have shown that one-variable pattern languages are learnable for basically all meaningful distributions within an optimal linear total learning time on the average. The algorithm obtained is quite simple and is based on symmetries that occur in such languages. Thus, our approach to minimize the expected total learning time turned out to be quite satisfactory.

Additionally, our learner requires only space that is linear in the length of the target pattern. Therefore, it is not only faster than the algorithms presented by Angluin [1] and Erlebach et al. [3] but also more spaceefficient. The only known algorithm using even less space is Lange and Wiehagen's [7] learner. But their algorithm is only successful for a much smaller class of probability distributions, since it requires shortest examples in order to converge. On the other hand, our learner maintains the incremental behavior of Lange and Wiehagen's [7] algorithm. While it is no longer iterative, it is still a bounded example memory learner. A learner is called iterative, if it uses only its last guess and the next example in the sequence of example strings for computing its actual hypothesis. A bounded example memory learner is additionally allowed to memorize an a priori bounded number of examples it already has had access to during the learning process. For more information concerning these learning models, we refer the reader to Lange and Zeugmann [8].

Moreover, our algorithm does not only possess an expected linear total learning time, but also very good tail bounds. Note that, whenever learning in the limit is considered one cannot decide whether or not the learner has already converged to a correct hypothesis. If convergence is decidable, we arrive at finite learning. It is easy to see that one-variable pattern languages are not finitely learnable. On the other hand, a bit of prior knowledge about the underlying probability distributions nicely buys a stochastically finite learner with high confidence. Recall that the number $G$ of rounds depends only on $p$ and $p_{\text {sym }}$. Now, assuming that one has the additional knowledge of upper bounds for both $p$ and $p_{\text {sym }}$, Formula 1 can be used to estimate the expected number of rounds. Let $\tilde{G}$ be this estimate, and let $\delta \in(0,1)$ be the confidence parameter given to the modified learner as additional input. Now, the modified learner computes the least $m$ such that $1-2^{m} \geq \delta$, and runs our algorithm for $2 \cdot m \cdot \tilde{G}$ rounds. While doing this, no output is provided. After having finished these rounds, the modified learner outputs the last guess $\pi$ made by our algorithm, and stops thereafter. Now, using the same argument as above for proving (2), one easily sees that $\pi$ must be correct for the target to be learned with probability at least $\delta$. Furthermore, the total learning time remains linear in the length of the target pattern.

Note that stochastically finite learning with high confidence is different from PAC-learning. First, it is not completely distribution independent. Thus, from 
that perspective, this variant is weaker than the PACmodel. But the hypothesis computed is probably exactly correct. Moreover, the learner receives exclusively positive data while the correctness of its hypothesis is measured with respect to all data. Hence, from that perspective, our model of stochastically finite learning with high confidence is stronger than the PAC-model.

Our approach also differs from U-learnability introduced by Muggleton [9]. First of all, our learner is fed positive examples only, while in Muggleton's [9] model, examples labeled with respect to their containment in the target language are provided. Next, we do not make any assumption concerning the distribution of the target patterns. Furthermore, we do not measure the expected total learning time with respect to a given class of distributions over the targets and a given class of distributions for the sampling process, but exclusively in dependence on the length of the target. Finally, we require exact learning and not approximately correct learning.

We have implemented the algorithm and the reader is referred to http://www.itheoi.mu-luebeck.de/pages/ reischuk/Algor/learn/LearnUnser.html for getting access to the resulting Java-applets.

Next, we shortly discuss possible directions of further research. An obvious extension would be to consider $k$-variable pattern languages for small fixed $k>$ 1. Already for $k=2$ the situation becomes considerably more complicated and requires additional tools.

Another direction to pursue would be to learn languages that are the union of at most $\ell$ one-variable pattern languages for some fixed $\ell$.

Finally, the approach presented in this paper seems to be quite suited to tolerate errors in the example data. Let us assume that there is some (small) probability $\epsilon$ that

error model 1: in an example string $X[1] \ldots X[l]$ a symbol $X[i]$ is changed to a different one,

error model 2: $\quad X[i]$ is changed to a different symbol or removed or replaced by two symbols $X[i] \sigma$ for some $\sigma \in \Sigma$.

A property of the pattern language like the common prefix of all strings now is only accepted if it is supported by a large percentage of examples. The details and modification of the algorithm will be given in another paper.

\section{References}

[1] D. Angluin. Finding Patterns Common to a Set of Strings, Journal of Computer and System Sciences 21:46-62, 1980.

[2] M. Crochmore and W. Rytter. Text Algorithms, Oxford University Press, 1994.

[3] T. Erlebach, P. Rossmanith, H. Stadtherr, A. Steger and T. Zeugmann. Efficient learning of onevariable pattern languages from positive data, DOITR-128, Kyushu University, Fukuoka, Japan, 1996.
[4] T. Erlebach, P. Rossmanith, H. Stadtherr, A. Steger and T. Zeugmann. Learning One-Variable Pattern Languages Very Efficiently on Average, in Parallel, and by Asking Queries, in M. Li and A. Maruoka (Eds.), Proc. 8th International Workshop on Algorithmic Learning Theory, 1997, Lecture Notes in Artificial Intelligence 1316, 260-276, Springer-Verlag.

[5] E. Gold. Language identification in the limit, Information \& Control 10:447-474, 1967.

[6] M. Kearns and L. Pitt. A polynomial-time algorithm for learning $k$-variable pattern languages from examples, in R. Rivest, D. Haussler and M. K. Warmuth (Eds.), Proc. 2nd Annual ACM Workshop on Computational Learning Theory, 1989, 57-71, Morgan Kaufmann.

[7] S. Lange and R. Wiehagen. Polynomial-time inference of arbitrary pattern languages, New Generation Computing 8:361-370, 1991.

[8] S. Lange and T. Zeugmann. Incremental Learning from Positive Data, Journal of Computer and System Sciences 53(1):88-103, 1996.

[9] S. Muggleton. Bayesian Inductive Logic Programming, in M. Warmuth (Ed.), Proc. 7th Annual ACM Conference on Computational Learning Theory, 1994, 3-11, ACM Press.

[10] L. Pitt. Inductive inference, DFAs and computational complexity, in K.P. Jantke (Ed.), Proc. 2nd International Workshop on Analogical and Inductive Inference, 1989, Lecture Notes in Artificial Intelligence 397, 18-44, Springer-Verlag.

[11] R. Reischuk and T. Zeugmann. Learning OneVariable Pattern Languages in Linear Average Time, DOI-TR-140, Kyushu University, Fukuoka, Japan, 1997. http://www.i.kyushu-u.ac.jp/ thomas/tr.html

[12] A. Salomaa. Patterns, (The Formal Language Theory Column), EATCS Bulletin 54:46-62, 1994.

[13] A. Salomaa. Return to patterns, (The Formal Language Theory Column), EATCS Bulletin 55:144157, 1994.

[14] R. Schapire. Pattern languages are not learnable, in M.A. Fulk and J. Case (Eds.), Proc. 3rd Annual ACM Workshop on Computational Learning Theory, 1990, 122-129, Morgan Kaufmann.

[15] T. Shinohara and S. Arikawa. Pattern inference, in "Algorithmic Learning for Knowledge-Based Systems," (K. Jantke and S. Lange (Eds.)) Lecture Notes in Artificial Intelligence 961, 1995, 259-291, Springer-Verlag.

[16] R. Wiehagen and T. Zeugmann. Ignoring data may be the only way to learn efficiently, Journal of Experimental and Theoretical Artificial Intelligence 6:131-144, 1994.

[17] T. Zeugmann. Lange and Wiehagen's pattern language learning algorithm: An average-case analysis with respect to its total learning time, RIFIS-TR 111, Kyushu University, Fukuoka, Japan, 1995, to appear in Annals of Mathematics and Artificial Intelligence. 